\title{
Temperature Dependence of Peak Positions in Pair Distribution Function of Metallic Liquids
}

W.B. Zhang ${ }^{\mathrm{a}}$, X.D. Wanga, ${ }^{\mathrm{a}}$, Q.P. Cao ${ }^{\mathrm{a}}$, D.X. Zhang, ${ }^{\mathrm{a}, \mathrm{b}}$, and J.Z. Jianga,

${ }^{a}$ International Center for New-Structured Materials (ICNSM), Laboratory of New-Structured Materials, State Key Laboratory of Silicon Materials, and School of Materials Science and Engineering, Zhejiang University, Hangzhou, 310027, People's Republic of China

${ }^{b}$ State Key Laboratory of Modern Optical Instrumentation, Zhejiang University, Hangzhou, 310027, People's Republic of China

*Authors to whom correspondence should be addressed: wangxd@zju.edu.cn and jiangjz@zju.edu.cn 

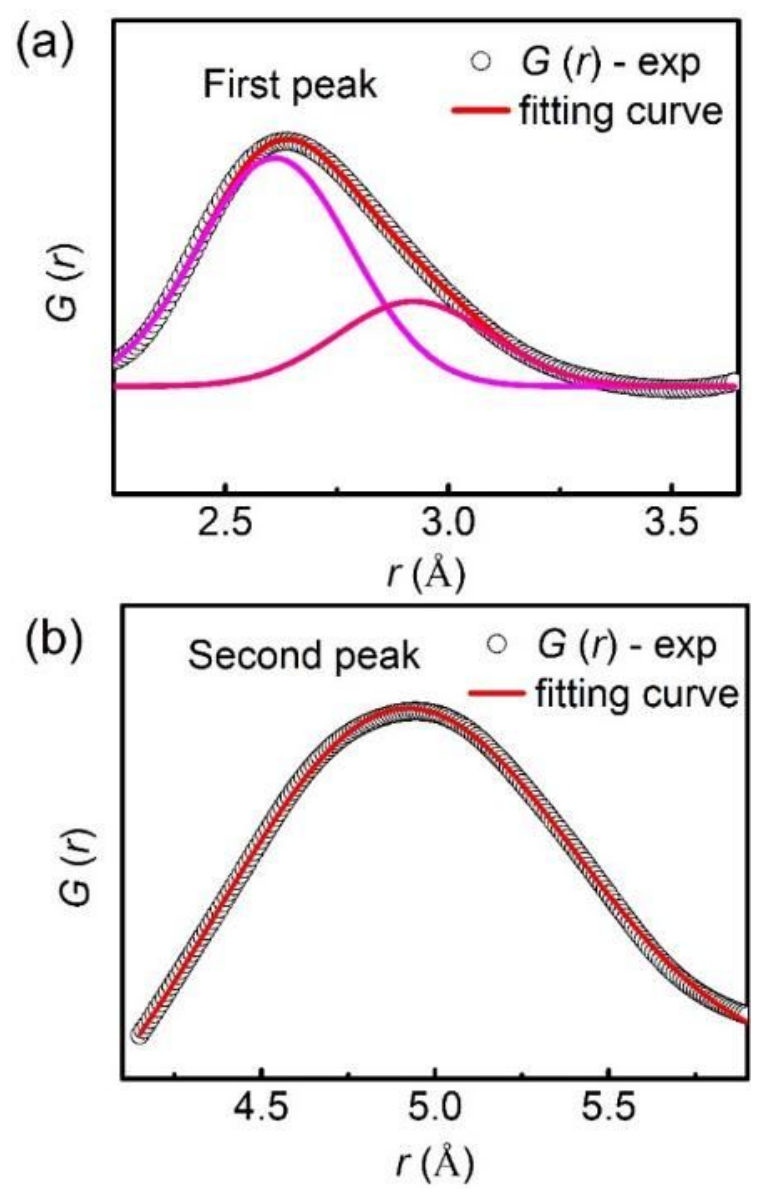

Figure. S1 (a) the fit (red curve) of the first peak of experimental pair distribution function $G(r)$ (black open circles) using two Gaussian functions (magenta and pink curves). (b) the fit (red curve) of the second peak of experimental pair distribution function $G(r)$ (black open circles) using one Gaussian function. 


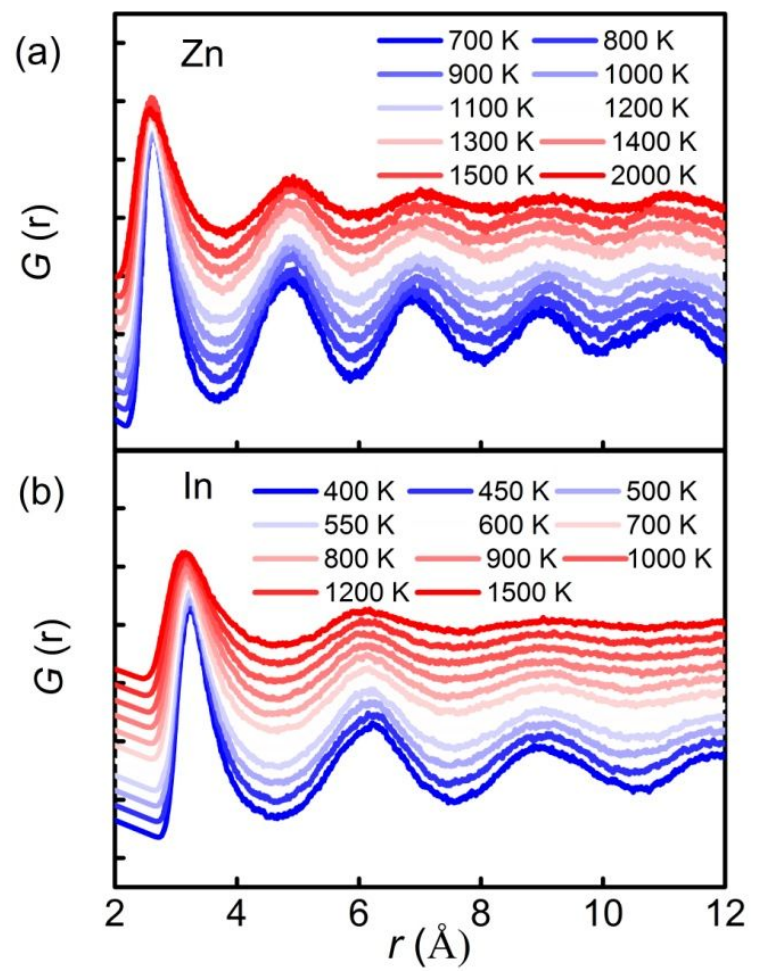

Figure. S2 Pair distribution functions $G(r)$, calculated from the AIMD configurations of (a) liquid $\mathrm{Zn}$ and (b) liquid In at different temperatures. 

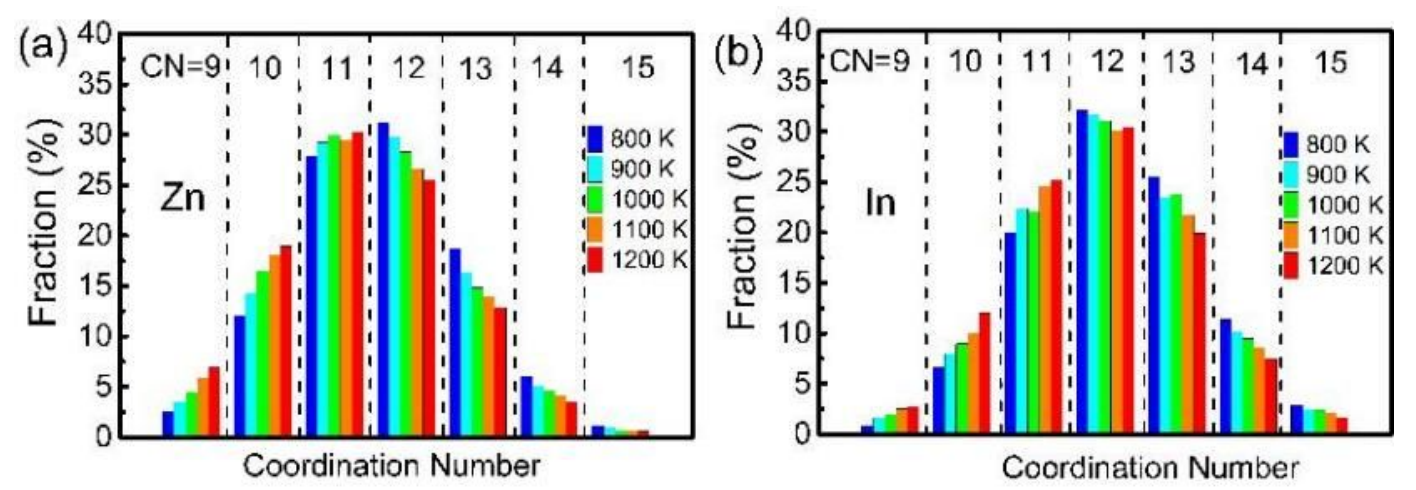

Figure. S3 Fractions of major polyhedra larger than $2 \%$ with different coordination numbers at 800, 900, 1000, 1100 and $1200 \mathrm{~K}$ for (a) liquid $\mathrm{Zn}$ and (b) liquid In, deduced by the Voronoi tessellation method from AIMD simulations. It should be noted that just for comparison, the fractions of $\mathrm{CN}=15$ polyhedra of $\mathrm{Zn}$ atoms smaller than $2 \%$ are also plotted. 


\section{SI text}

\section{Simulation details}

Ab initio molecular dynamics simulations of liquid $\mathrm{Zn}$ and In were performed using Vienna $a b$ initio simulation package (VASP). ${ }^{1}$ The interactions between electrons and ions were described by a Projector Augmented-Wave (PAW) method. ${ }^{2}$ The general gradient approximation (GGA) in the form of Pedrew-Burke-Ernzerh (PBE) was used for the exchange-correlation energy functional. ${ }^{3} \mathrm{All}$ MD simulations were carried out in a NVT (i.e. constant atom number, volume, and temperature) ensemble with the temperature controlled by Nose's Hoover thermostat. ${ }^{4}$ A cubic supercell containing $120 \mathrm{Zn}$ atoms was melted at $2000 \mathrm{~K}$ and relaxed for $30 \mathrm{ps}$ (each step time 3 fs) to eliminate the memory effect of the initial configuration and allow the system to reach a relatively equilibrium state. Then, the temperature was stepwise lowered to $1500,1400,1300,1200,1100,1000,900,800,700 \mathrm{~K}$ at a cooling rate of $0.1 \mathrm{~K} /$ step. For liquid In, the cubic cell of $120 \mathrm{In}$ atoms was initially melted at $1500 \mathrm{~K}$, then cooled to $1200,1100,1000,900,800,700,600,550,500,450 \mathrm{~K}$ in steps. At each temperature, the system was relaxed for more than 18 ps to ensure the equilibrium. The volume of the supercell was adjusted according to the internal pressure with a criterion about \pm $0.5 \mathrm{kbar}$. The configurations at last 9 ps were selected for structure statistical analyses.

\section{Voronoi tessellation}

Using the Voronoi tessellation method, ${ }^{5}$ the fraction changes of various dominant polyhedra $(>2 \%)$ in $\mathrm{Zn}$ and In liquids with coordination numbers from $\mathrm{CN}=9$ to 15 are showed in Figs. S3 (a) and (b) at different temperatures from 800 to $1200 \mathrm{~K}$, respectively. It is found that in the liquid $\mathrm{Zn} \mathrm{CN}=11$ and 12 coordinated polyhedra have similar fractions about $25-30 \%$, while only $\mathrm{CN}=12$-coordinated polyhedra is dominant for more than $30 \%$ in the liquid In. By comparing with liquid $\mathrm{Zn}$, the liquid In has more large $\mathrm{CN}=13$ and 14-coordinated polyhedra but less $\mathrm{CN}=9,10,11$-coordinated ones. The average coordination numbers for In and $\mathrm{Zn}$ are found to be_12.15 and 11.70 at 800 $\mathrm{K}$, and 11.75 and 11.16 at $1200 \mathrm{~K}$, respectively. It is clear that for both liquids, the fractions for small $\mathrm{CN}=9,10,11$-coordinated polyhedra increase while they for large 
$\mathrm{CN}=12,13,14,15$-coordinated polyhedra decrease when temperature increases from

800 to $1200 \mathrm{~K}$. And the result of Voronoi tessellation indicate that the first peak shifts to low $r$ direction with temperature within the SRO is resulted from the evolution from large-sized polyhedra to small ones as reported in Ref. 6.

\section{References:}

(1) Kresse, G.; Furthmuller, J. Efficiency of ab-initio total energy calculations for metals and semiconductors using a plane-wave basis set. Comput. Mater. Sci. 1996, 6, 15-50.

(2) Blöchl, P. E. Projector augmented-wave method. Phys. Rev. B 1994, 50, 17953.

(3) Perdew, J.P.; Burke, K.; Ernzerhof, M. Generalized gradient approximation made simple. Phys. Rev. Lett. 1996, 77, 3865.

(4) Nose, S. A unified formulation of the constant temperature molecular dynamics methods. J. Chem. Phys. 1984, 81, 511-519.

(5) Finney, J. L. Modeling structure of amorphous metals and alloys. Nature. 1977, 266, 309-314.

(6) Lou, H.; Wang, X.; Cao, Q.; Zhang, D.; Zhang, J.; Hu, T.; Mao, H.K.; Jiang, J.Z. Negative expansions of interatomic distances in metallic melts. Proc. Natl. Acad. Sci. U. S. A. 2013, 110, 10068. 